\title{
Traveling liquid bridges in unsaturated fractured porous media
}

\author{
Dani Or • Teamrat A. Ghezzehei
}

Received: 22 June 2005 / Accepted: 25 January 2006 / Published online: 3 November 2006

(C) Springer Science+Business Media B.V. 2006

\begin{abstract}
Interplay between capillary, gravity and viscous forces in unsaturated fractures gives rise to a range of complex flow phenomena. Evidence of highly intermittent fluxes, preferential and sustainable flow pathways lead to potentially significant flow focusing of concern for regulatory and management of water resources in fractured rock formations. In previous work [Ghezzehei TA,Or D.: Water Resour. Res. In Review(2005)] we developed mechanistic models for formation, growth and detachment of liquid bridges in geometrical irregularities within fractures. Such discrete and intermittent flows present a challenge to standard continuum theories. Our focus here is on predicting travel velocities of detached liquid elements and their interactions with fracture walls. The scaling relationships proposed by Podgorski et al. [Podgorski, T., et al.: Phys. Rev. Lett. 8703(3), 6102-NIL_95 (2001)] provide a general framework for processes affecting travel velocities of discrete liquid elements in fractures, tubes, and in coarse porous media. Comparison of travel velocity and distance by discrete bridges relative to equivalent continuous film flow reveal significantly faster and considerably larger distances traversed by liquid bridges relative to liquid films. Coalescence and interactions between liquid bridges result in complex patterns of travel times and distances. Mass loss on rough fracture surfaces shortens travel distances of an element; however, results show that such retardation provides new opportunities for coalescence of subsequent liquid elements traveling along the same path, resulting in mass accumulation and formation of larger liquid elements traveling larger distances relative to smooth fracture surfaces. Such flow focusing processes may be amplified considering a population of liquid bridges within a fracture plane and mass accumulation in fracture intersections.
\end{abstract}

D. Or $(\bowtie)$

School of Architectural, Civil and Environmental Engineering (ENAC/ISTE),

Ecole Polytechnique Federale de Lausanne (EPFL), Bâtiment, GR 2 (room 399),

CH-1015, Lausanne, Switzerland

e-mail: dani.or@epfl.ch

T. A. Ghezzehei

Earth Sciences Division, Lawrence Berkley National Laboratory, Berkley, CA, USA 
Keywords Intermittent flow $\cdot$ Fracture $\cdot$ Dripping $\cdot$ Liquid bridge $\cdot$ Transport

\section{Introduction}

Recent field measurements of infiltration and flow in unsaturated fractured rocks revealed highly intermittent fluxes emerging from fractures (Faybishenko et al. 2003, 2000). These findings are supported by laboratory visualization studies in fracture analogs (Ghezzehei and Or 2005; Nicholl et al. 1994; Persoff and Pruess 1995; Su et al. 1999, 2004) that implicate the formation and detachment of discrete liquid elements (also referred to as liquid bridges) within geometrical constrictions, intersections, and other irregularities on surfaces of unsaturated fractures. These resulting flow regimes were described by Faybishenko et al. (2000) as follows: "in the temporal regime we recognize that flow through an unsaturated fracture network may be more like a set of dripping faucets than the continuous flow predicted by Darcy's law and Richards' equation".

Several mechanistic and percolation-based models have been proposed for the onset and dynamics of liquid bridge growth and detachment in idealized fracture geometries (Ghezzehei and Or 2005; Glass et al. 2002; Ho 2004); however, little attention was given to the potentially important transport pathway associated with rapidly moving detached liquid clusters of considerable size through fractured formations that would otherwise be considered hydraulically inactive. Data show that under certain conditions (e.g., vertical rough-walled fractures and film flow), liquid bridges that attain a certain size overcome pinning capillary forces and traverse large distances in a short time carrying with them dissolved substances and result in significant intermittent fluxes even in the absence of hydrologic continuity. For constant flux and ambient conditions the process may be periodic and predictable (Ghezzehei and Or 2005), however, the detachment dynamics and subsequent motions of such discrete liquid elements are not amenable to continuum representation using classical equations of unsaturated flow in porous media.

The general objective of this study is to develop mechanistic models for the motion of detached liquid bridges and discuss consequences of such motion on transport potential and fluxes within unsaturated fractured rock. Specifically, to: (1) develop models for liquid bridge geometry and travel velocity; (2) incorporate restraining forces affecting travel velocity and mass loss (roughness streaking and matrix imbibition); (3) study interactions between multiple bridges and onset of cascading avalanches; and (4) integration of the above to description of macroscopic transport potential and flux intermittency.

In the following, we present a brief recap of liquid bridge formation and detachment. The transition from stationary to sliding bridge obeys general scaling law that combines capillary, viscous, and gravitational forces. Applications for various conditions require considerations of bridge size and shape affecting energy dissipation and restraining viscous forces. The theoretical section will conclude with consideration of mass exchange with rock matrix and fracture surface, and coalescence with other bridges in the path forming an avalanche. The theoretical results will be illustrated using available experimental data.

\section{Theoretical considerations}

The travel of discrete liquid bridges in unsaturated fractures bears strong resemblance to motion of liquid slugs in capillary tubes studied by Bico and Quere (2001), 
and the sliding of drops along inclined surfaces (Hoffman 1975; Kim et al. 2002; Podgorski et al. 2001). The development of mechanistic models in this section involves adaptation of concepts and approximations successfully used previously in modeling these analog problems. To facilitate the mathematical derivations that follow, we make the following simplifying assumptions regarding fracture geometry and characteristics.

1. Smooth and impervious (the latter aspect will be revised at a later stage),

2. Parallel walled narrow aperture fractures.

The section is arranged as follows: 2.1 provides a summary of our previous work on formation of liquid bridges; 2.2 addresses the geometry and conditions for stationary bridges at the onset of motion limit; in 2.3 a general framework and specific expressions for the steady velocity of traveling liquid bridges are developed; finally, in 2.4 we address interactions among traveling bridges and with fracture walls giving rise to complex mass-travel patterns.

\subsection{Formation and detachment of liquid bridges (Ghezzehei and Or 2005)}

In this subsection, we provide a brief recap of main results from our recent study on modeling liquid bridge formation and detachment in unsaturated fractures (Ghezzehei and Or 2005). The formation of liquid bridges is associated with asperities and other geometrical perturbations that provide anchors for capillary pinning of a growing liquid bridge (Su et al. 2004, Wood et al. 2004). The cyclical process of growth and discharge of liquid bridges is perpetuated by the presence of sufficient liquid flux diverted towards an anchoring spot in the fracture (either as film or matrix flow). The setting and process are idealized in the geometrical model shown in Fig. 1, which considers a fracture of uniform aperture $b$ inclined at an angle of $\alpha$ from a horizontal plane. A constant volumetric flow rate $Q$ is diverted towards the obstruction forming a liquid bridge beneath it. The model estimates the weight of the discharged liquidmass and discharge rate (periodicity) as functions of geometry, flow rate, and fluid properties.

Considering, for simplicity, an initially semi-circular liquid bridge growing in the fracture plane, it is useful to define the dimensionless Bond number $(\mathrm{Bo})$ reflecting the importance of gravitational relative to capillary forces acting on the liquid mass:

$$
\mathrm{Bo}=\frac{\rho g b y^{2} \sin \alpha}{2 \sigma(2 b-y \cos \theta)}
$$

where $y$ is the width of the liquid bridge and $\theta$ is contact angle. When the lateral extent of the liquid mass is much greater than the fracture aperture $(y \gg b)$, the Bond number grows with the diameter of the liquid mass $y$ and/or the inclination angle $\alpha$. The liquid responds to large Bond number (excessive gravitational forces compared to capillary forces) by adjusting the advancing and receding contact angles and assuming a non-circular (elongated) geometry. For simplicity, we assume existence of a threshold Bond number Bo*, which marks the onset of elongation. We also assume that subsequent elongation occurs at a fixed threshold diameter, $y_{0}$, given by rearranging (1) 


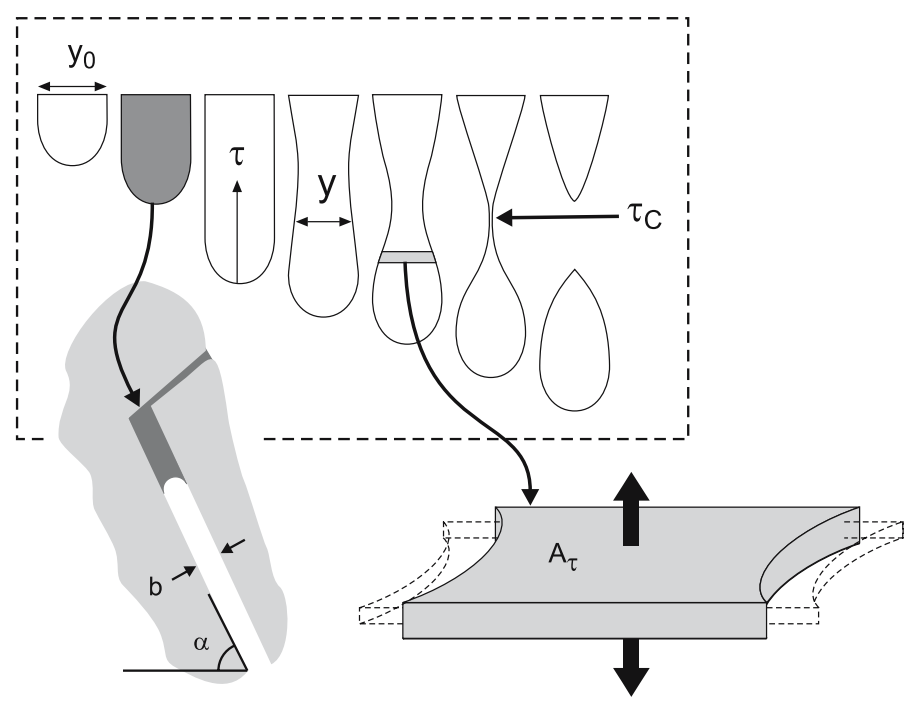

Fig. 1 Idealized representation of liquid bridge suspended below fracture discontinuity and schematic illustration of elongation and extension of a typical fluid element

$$
y_{0}=\frac{\mathrm{Bo}^{*} \sigma}{\rho g b \sin \alpha}\left(\frac{\cos \theta}{b}+\sqrt{\cos ^{2} \theta+\frac{\rho g \sin \alpha}{\mathrm{Bo}^{*} \sigma} b^{2}}\right)
$$

The above two assumptions were supported by observations (Ghezzehei and Or 2005), yielding an estimate of the critical Bond number $\mathrm{Bo}^{*}=0.05$. A similar value of Bo* (0.0125-0.033) was observed in experiments by Prazak et al. (1992) as giving rise to intermittent flow regime in packs of coarse sand.

Liquid addition after onset of elongation results in an increase in bridge length at relatively uniform and constant width as long as interfacial (capillary) forces remain larger than the weight of the suspended liquid bridge. At a certain critical value of suspended bridge mass, a narrowing neck appears leading to stretching and eventually a complete detachment of the liquid below the neck. These distinct stages of growth and detachment are illustrated in Fig. 1.

The elongation and subsequent breakup are modeled as a modified one-dimensional extensional flow (Wilson 1988). Neglecting inertial effects, an elongating liquid bridge of known cross-boundary sectional area $A_{0}$ and fed by a constant volumetric flow rate $Q$, the cross section of the bridge at any position $\mathrm{z}$ is given by

$$
3 \eta \frac{\partial A(z, t)}{\partial t}=\rho g V(z, t)
$$

where $A(z, t) \approx 2 b y(z, t)$ is the cross sectional area of the liquid bridge at $z$ and $V(z, t)$ is the volume suspended below $z$. To facilitate derivation of analytical solution to the problem we switch from the above fixed Cartesian coordinates to moving (Lagrangian) one-dimensional coordinate (Wilson 1988). An arbitrary fluid element located at some $z$ is marked by the time of its departure from the extrusion plane $t=\tau$. It follows then that $A(z, t)$ is equivalent to $A(\tau, t)$. Moreover, the constant rate of liquid supply leads to equivalence of the volume $V(z, t)$ with $Q \cdot \tau$. For liquid bridges confined within narrow fractures two additional resistive force operate. First, the advancing bridge tip 
is met with capillarity resistive pressure of the order of $\Phi=2 \sigma \beta \mathrm{Ca}^{2 / 3} / b$ (Bico and Quere 2001). Secondly, surface tension of the liquid-vapor interface resists shrinkage of the cross-sectional area due to a force of the form $\Psi=2 \sigma b(\pi-2 \theta) / \cos \theta$. Then, the full equation of extension for a liquid bridge growing within a fracture is given as

$$
2 b \lambda \frac{\partial y(\tau, t)}{\partial t}=\rho g Q \tau \sin \alpha-2 b \Phi y(\tau, t)-\Psi
$$

where $\lambda=3 \eta$ is the coefficient of extensional viscosity. The solution to (4) subject to the boundary condition $y_{0}=y_{\tau}(t=\tau)$ (constant liquid bridge width at the top boundary obtained using (2)) is

$$
\frac{\Phi}{\lambda} y_{\tau}(t)=\frac{\rho g Q \tau \sin \alpha-\Psi}{2 b \lambda}-\left\{\frac{\rho g Q \tau \sin \alpha-\Psi}{2 b \lambda}-\frac{\Phi}{\lambda} y_{0}\right\} \exp \left(\frac{\Phi}{\lambda}(\tau-t)\right)
$$

Equation 5 describes the evolution of the width of a typical fluid element $\tau$. When the liquid bridge cannot support its own weight and a narrow neck forms and eventually raptures releasing a freely sliding liquid mass as shown in Fig. 1. During detachment, the following two conditions occur at the rapture plane. First, the width of the critical fluid element $\tau=\tau_{\mathrm{C}}$ vanishes at some time $t=t^{*}$

$$
t^{*}=\tau_{\mathrm{C}}+\frac{\lambda}{\Phi} \ln \left[1-\frac{2 b y_{0} \Phi}{\rho g Q \tau_{\mathrm{C}} \sin \alpha-\Psi}\right]
$$

Secondly, the rate of extension of the liquid forming the rupturing neck increases at a very rapid rate $\mathrm{d} \tau_{\mathrm{C}} / d \tau_{\mathrm{C}} \rightarrow \infty$ leading to an expression for the time interval between two consecutive detachment events

$$
\rho Q \tau_{\mathrm{C}}=\frac{\Psi}{g \sin \alpha}+\frac{b y_{0} \Phi}{g \sin \alpha}\left\{1+\sqrt{1-2 \frac{\rho g Q \lambda}{b y_{0} \Phi^{2}}}\right\}
$$

Accordingly, the mass of detached liquid bridge is $M_{\mathrm{C}}=\rho Q \tau_{\mathrm{C}}$. The first term on the right-hand-side of (7) is indicative of the static mass supported by the surface tension of the liquid-vapor interface. The second term represents the mass supported by the dynamic contact angle (result of flow and presence of fracture walls). Subsequent analysis showed that the dynamic part of the solution does not play a significant role in determining detachment mass, resulting in a simple (and more practical) expression for the liquid bridge mass:

$$
M_{\mathrm{C}}=\frac{2 \sigma(\pi-2 \theta)}{\sin \alpha \cos \theta g} b
$$

\subsection{From stationary to sliding liquid bridges}

Seemingly different approaches to modeling transition from stationary to sliding drops and discrete liquid bridges (in the presence of retarding capillary interactions with solid surfaces and capillary spaces) lead to a surprisingly simple and general scaling law proposed by Podgorski et al. (2001). Without involving the exact shape of a liquid bridge and details of associated flow field, Podgorski et al. (2001) proposed the following:

$$
\mathrm{Ca} \sim \mathrm{Bo} \sin \alpha-\Delta_{\theta}
$$


where $\mathrm{Ca}$ is the Capillary number $\mathrm{Ca}=\eta U / \sigma$ ( $U$ is bridge sliding velocity); $\mathrm{Bo}$ is the Bond number defined generically as $\mathrm{Bo}=V^{2 / 3} \rho g / \sigma$ ( $V$ is the bridge volume); and $\Delta_{\theta}$ is a perimeter-averaged projection of surface tension (Extrand and Kumagai 1995), which may also be viewed as the minimum value of $B o \sin \alpha$ below which the bridge remains pinned by capillary forces. For Bo larger than a threshold value, the capillary number increases linearly with $B o \sin \alpha$ until it attains a velocity that results in pearling and disintegration of the liquid bridge (Podgorski et al. 2001). In Appendix 1 , we provide an alternative derivation of the scaling law based on general energy dissipation equations.

The general conditions and geometries controlling the threshold value $\Delta_{\theta}$ are explored next considering a simple one-dimensional formulation of liquid bridge dynamics, followed by more realistic (and complicated) two-dimensional formulation (assuming elliptical liquid bridges).

\subsubsection{One-dimensional analysis}

Consider a liquid bridge of length $L$ held in an idealized fracture described above (Fig. 2). We derive an expression for the threshold mass that triggers motion from considerations of (a) force balance and (b) hydrostatic pressure balance.

Force balance: The component of the liquid bridge weight that pulls the bridge downward is balanced by the net sum of the surface tension forces at the advancing and receding edges:

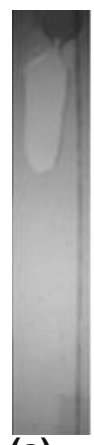

(a)
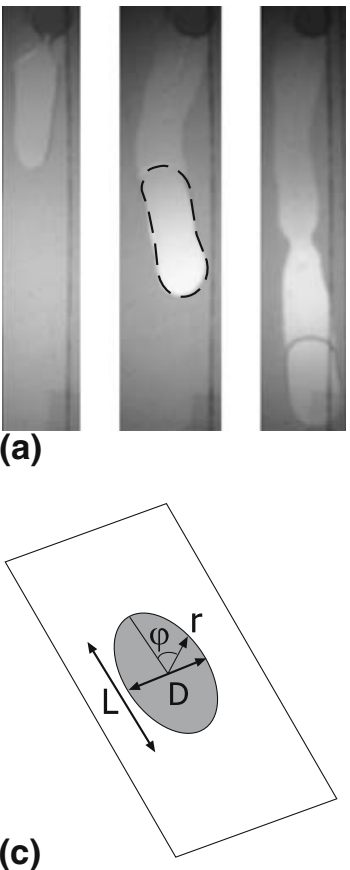

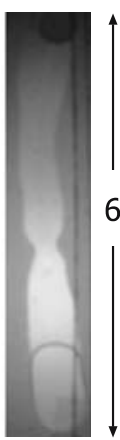

$65 \mathrm{~mm}$

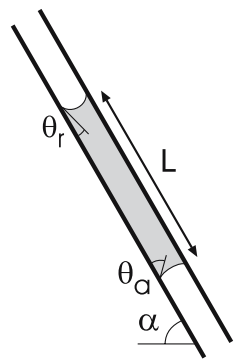

(b)

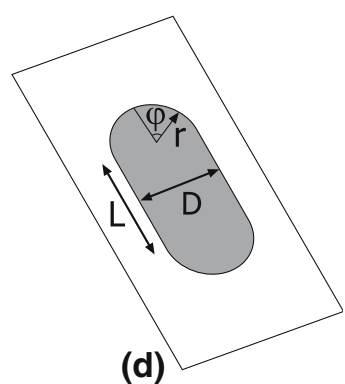

Fig. 2 (a) Liquid bridges sliding down a parallel-glass fracture model of $b=0.2 \mathrm{~mm}$ aperture, (b) onedimensional conceptual model of liquid bridges, and (c) elliptical (two-dimensional) conceptual model of liquid bridges, (d) rectangular center with circular advancing and receding edges (see Appendix A) 


$$
V \rho g \sin \alpha=2 \mathrm{~d} W \sigma\left(\cos \theta_{\mathrm{r}}-\cos \theta_{\mathrm{a}}\right)
$$

where $\theta_{\mathrm{r}}$ and $\theta_{\mathrm{a}}$ are the receding and advancing contact angles, respectively; $\mathrm{d} W$ is unit width of the liquid bridge, and the bridge volume is $V=\mathrm{d} W \cdot L b$. The factor of two in the right hand side of Eq. 10 accounts for the two walls of the fracture.

Pressure balance: We assume that the fluid forming a stationary liquid bridge is at rest, satisfying a uniform hydrostatic pressure everywhere. Consequently, the difference in capillary pressure $(\psi)$ between the advancing and receding menisci is offset by the gravitational head difference:

$$
\psi_{\mathrm{a}}-\psi_{\mathrm{r}}=\rho g L \sin \alpha
$$

The capillary pressure of the menisci is related to the aperture and contact angle by Laplace-Young equation

$$
\psi=-2 \cos \theta \sigma / b
$$

The two approaches above (Eqs. 10,11) can be reduced to identical dimensionless scaling law

$$
\frac{\rho g b L}{2 \sigma} \sin \alpha=\left(\cos \theta_{\mathrm{r}}-\cos \theta_{\mathrm{a}}\right)
$$

Comparison of (13) with the scaling law of Podgorski et al. (2001) given by (9) reveals that the critical Bo $\sin \alpha$ required for onset of motion is

$$
\frac{\rho g b L_{\mathrm{C}}}{2 \sigma} \sin \alpha=\left(\cos \theta_{\mathrm{r}}^{*}-\cos \theta_{\mathrm{a}}^{*}\right)=\Delta_{\theta}
$$

where $L_{\mathrm{C}}$ is the minimum liquid bridge length required for the onset of motion, and $\theta_{\mathrm{r}}$ and $\theta_{\mathrm{a}}$ are the critical receding and advancing contact angles, respectively. According to (13) the onset of motion is determined by bridge length and contact angle hysteresis (bridge width plays no role in this 1-D analysis).

\subsubsection{Two-dimensional analysis}

Consider a liquid bridge whose size in the fracture plane is much larger than the fracture aperture such that the contribution of in-plane curvature to total interfacial curvature (hence, pressure difference across the liquid-vapor interface) can be ignored. The geometric variables are described in Fig. 2. In radial coordinates, the bridge perimeter is defined by

$$
r=\frac{L \lambda}{2 \sqrt{\sin ^{2} \varphi+\lambda^{2} \cos ^{2} \varphi}}
$$

where $L$ is the major diameter (length) of the bridge, $\lambda=D / L$ is the aspect ratio, and $0 \leq \varphi \leq 2 \pi$ is angle measured clock-wise from the receding tip. Assuming uniform total pressure, the capillary pressure linearly changes with elevation as

$$
\psi=\psi_{0}+h
$$

At the mid-point $(\varphi=\pi / 3)$, the stress on the solid-liquid-vapor contact line due to the bridge weight vanishes; thus, the contact angle does not deviate from the static contact angle value of $\theta_{0}$. Then, according to Eq. $12, \psi_{0}=-2 \cos \theta_{0} / b$. Accordingly, 
the elevation of any point along the perimeter of the bridge with respect to the mid-point is given by

$$
h=r \cos \varphi \sin \alpha
$$

The local contact angle along the perimeter adjusts to maintain equilibrium hydrostatic pressure:

$$
\psi=-\frac{2 \sigma}{b} \cos \theta=\psi_{0}+h
$$

Rearranging Eq. 18 gives

$$
\cos \theta=\cos \theta_{0}-\frac{\gamma}{4} \frac{\lambda \cos \varphi}{\sqrt{\lambda^{2} \cos ^{2} \varphi+\sin ^{2} \varphi}}
$$

We note that Extrand and Kumagai (1995) arrive at a slightly different expression (for a stationary elliptical drop on inclined plate) by assuming a linear increase in $\cos \theta$ with $\varphi$, an assumption that does not satisfy the capillary pressure equilibrium. Along an infinitesimal segment of the perimeter $\mathrm{d} S$, surface tension pulls the edges of the liquid bridge outward (normal to the contact line) by a force equivalent to $2 \sigma \cos \theta \mathrm{d} S$. Note that a factor of two is applied to represent both fracture walls. The component of this force acting parallel to the major axis (opposing the weight) is

$$
\mathrm{d} F_{S}=2 \sigma \cos \theta \cos \varphi \mathrm{d} S
$$

and the length of the infinitesimal segment is (Spiegel and Liu 1999)

$$
\mathrm{d} S=\frac{L}{2} \sqrt{1+\sin ^{2} \varphi\left(\lambda^{2}-1\right)} \mathrm{d} \varphi
$$

Then, the total force supporting the sliding weight of the bridge is given by integrating the force along the perimeter of the bridge (note that the integration is applied for a semi-ellipse and a factor of two is used to account for the full ellipse)

$$
\begin{gathered}
F_{U}=-4 \sigma L \int_{0}^{\pi} \cos \theta \cos \varphi \sqrt{1+\sin ^{2} \varphi\left(\lambda^{2}-1\right)} \mathrm{d} \varphi=L \sigma_{L V} \gamma \lambda f(\lambda) \\
f(\lambda)=\int_{0}^{\pi} \cos \varphi^{2} \frac{\sqrt{\cos ^{2} \varphi+\lambda^{2} \sin ^{2} \varphi}}{\sqrt{\lambda^{2} \cos ^{2} \varphi+\sin ^{2} \varphi}} \mathrm{d} \varphi
\end{gathered}
$$

The component of the bridge weight acting along the fracture plane is approximately (ignoring the contribution of edge meniscus) given by

$$
F_{W}=\frac{\pi b L^{2} \rho g \lambda}{4} \sin \alpha
$$

For the liquid bridge to remain stationary, the sum of the forces has to vanish. Setting the sum of (22) and (24) to zero and rearranging gives (Fig. 3),

$$
2 F_{U}+F_{W}=0=\frac{\pi}{2}-f(\lambda)
$$

Note that Eq. 25 depends only on the aspect ratio $\lambda=D / L$. Numerical evaluation of (25) indicates that $\lambda=1$; i.e., circular geometry is energetically the most preferred Springer 


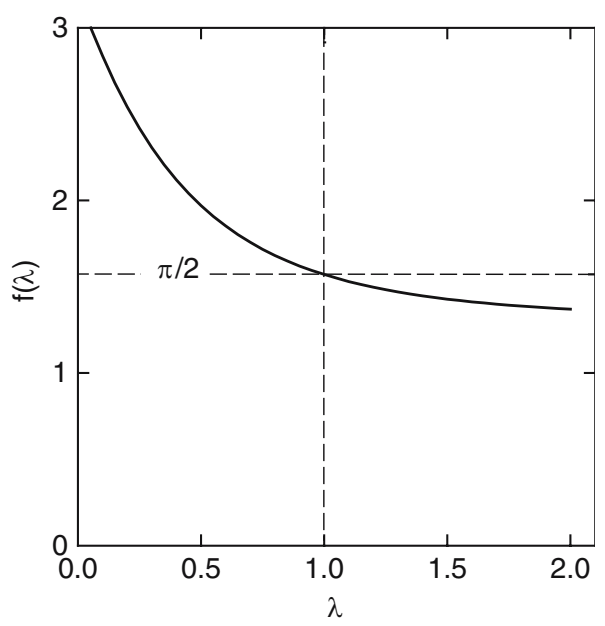

Fig. 3 Numerical evaluation of $f(\lambda)$

for stationary liquid bridge. Substituting $\lambda=1$ in Eq. 19 and evaluating it at the advancing $\left(\varphi=\pi\right.$ and $\left.\theta=\theta_{\mathrm{a}}\right)$ and receding $\left(\varphi=0\right.$ and $\left.\theta=\theta_{\mathrm{r}}\right)$ tips leads to

$$
\frac{\rho g b L}{2 \sigma} \sin \alpha=\operatorname{Bo} \sin \alpha=\left(\cos \theta_{\mathrm{r}}-\cos \theta_{\mathrm{a}}\right)
$$

This is an identical result to the scaling law (13) derived using one-dimensional derivation. The result suggests that stationary liquid bridges tend towards a circular shape.

\subsection{Steady motion of liquid bridges}

In this section, we apply the scaling law of Podgorski et al. (2001) (given by Eq. 9) to derive travel velocity of liquid bridges under various conditions. The scaling law unifies various concepts and analyses including the falling liquid slugs (Bico and Quere 2001) and motion of simple liquid bridges ( $\mathrm{Su}$ et al. 2004), and more. To extend the applicability of the scaling law to the dynamics of discrete liquid elements within a range of geometries (parallel plates, cylindrical tube, and porous media), we expand the definition of the Capillary number to (Meheust et al., 2002)

$$
\mathrm{Ca}=\frac{\eta U}{\sigma} \frac{a^{2}}{k}
$$

where $k$ is the permeability of the conduit where a liquid element forms and travels and $a$ is a characteristic length (fracture aperture, tube radius, or mean particle/pore size).

Consider the primary forces operating on a rectangular (1-D analysis above) liquid bridge in motion within a smooth parallel-wall fracture (Su et al. 2004) as shown in Fig. 2. For steady velocity, we balance viscous, capillary, and gravitational forces per unit width of a rectangular bridge as

$$
U L \eta \frac{12}{b^{2}}=\frac{\sigma}{b}\left(\cos \theta_{\mathrm{r}}^{*}-\cos \theta_{\mathrm{a}}^{*}\right)+\rho g L \sin \alpha
$$


Substituting the definition of the permeability for fluid flow between parallel plates $k=b^{2} / 12$ in (28) and rearranging reduces to

$$
\frac{\eta U}{\sigma} \frac{L b}{k}=\frac{\rho g L b}{\sigma} \sin \alpha-\Delta_{\theta}
$$

Note that Eq. 29 conforms to the scaling law of Podgorski et al. (2001) (Eq. 9). It can be shown that the steady velocity of slugs in capillary tubes (Bico and Quere 2001) and unstable finger flows in porous media (Meheust et al. 2002) are also reducible to the scaling law of Podgorski et al. (2001). The final expression differs only by the capillary pinning force geometry, characteristic lengths, and the permeability $k$ ( $k=r^{2} / 8$ for a tube; and in porous media $k \approx D^{2} / 700$, where $D$ is mean particle diameter). The scaling law can be stated as follows: the gravitational force in excess of capillary pinning force scales linearly with (or is proportional to) the viscous force. The scaling law can be used to estimate the capillary pinning forces without imposing any constraints on the shape of the discrete liquid masses (liquid bridges, slugs or fingers).

Equation 29 can be further reduced using the relation given by (14)

$$
\frac{U}{U_{\max }}=1-L_{\mathrm{C}} / L, \quad \text { for } L>L_{\mathrm{C}}
$$

where $U_{\max }=k \rho g \sin \alpha / \eta$ is the maximum flow velocity of a fully saturated fracture. Equation 30 describes the velocity of discrete sliding liquid bridge relative to the saturated hydraulic conductivity.

\subsection{Transient motion of liquid bridges}

The steady velocity of a liquid bridge described by (30) implies that once the liquid is released from its source (e.g., asperity) it remains intact until it encounters the fracture edge, an asperity, or another liquid bridge. The assumption is appropriate for smooth fractures embedded within relatively impervious matrix.

In this subsection, we extend the analysis by considering rough and/or porous fracture surfaces capable of retaining part of a traveling liquid bridge. Specifically, we evaluate the impact of loss of mass on the velocity of a traveling liquid bridge. The volume of liquid retained by surface depressions (roughness) or exposed matrix pores during an infinitesimal displacement of $\mathrm{d} x$ is related to the decrease in liquid bridge length according to,

$$
\mathrm{d} x \zeta=-\mathrm{d} L b
$$

where $\zeta$ denotes the volume of surface depressions and/or exposed pores per unit nominal fracture surface area. In the remainder of this paper, this property of fracture surfaces is referred to as "surface porosity". In Eq. 30, it is implied that the width of the liquid bridge does not change as mass is lost. Substituting (30) in (31) (where $\mathrm{d} x / \mathrm{d} t=U$ yields,

$$
\frac{\mathrm{d} L}{\mathrm{~d} t}=-K_{S} \frac{\zeta}{b}\left(1-\frac{L_{\mathrm{C}}}{L}\right)
$$

The solution to the ordinary differential equation (31) subject to the initial condition $L(t=0)=L_{0}$ is, 


$$
\frac{L(t)}{L_{\mathrm{C}}}=1+\operatorname{Product} \log \left[\left(\frac{L_{0}}{L_{\mathrm{C}}}-1\right) \exp \left(\left(\frac{L_{0}}{L_{\mathrm{C}}}-1\right)-\frac{K_{S} \zeta}{b L_{\mathrm{C}}} t\right)\right]
$$

The length of the traveling bridge decreases from its initial value $L=L_{0}$, and asymptotically approaches $L=L_{\mathrm{C}}$. The decrease of relative bridge length $L / L_{\mathrm{C}}$ is a function of elapsed travel time and fracture surface roughness $\zeta$. The decrease rate is faster for rougher surfaces. The distance traveled by the moving liquid bridge is given by integrating the velocity,

$$
S(t)=\int_{0}^{t} U(s) \mathrm{d} s=\frac{b L_{\mathrm{C}}}{\zeta} L(t)
$$

The initial condition $L_{0}=L(t=0)$ for liquid bridges emerging from an asperity is given by the liquid bridge at detachment (8),

$$
t=0, \quad L_{0}=\frac{M_{\mathrm{C}}}{\rho b y_{0}}=\frac{2 \sigma(\pi-2 \theta)}{\rho y_{0} \sin \alpha \cos \theta}
$$

Following coalescence with stalled liquid elements in a path of a moving liquid bridge, the resulting velocity of the combined element is faster and their combined weight (length) serves as a new initial condition. Consider a moving liquid bridge of length of $L\left(t=t^{*}\right)=L^{*}$ at the time of coalescence, and noting that the stalled bridge must have reached equilibrium length when it ceased to move (i.e., $L=L_{\mathrm{C}}$ ) the initial condition at the time of restart for liquid bridge formed by coalescence of moving liquid bridge with a stationary one is given as,

$$
t=0, \quad L_{0}=L_{\mathrm{C}}+L^{*}
$$

The exact mechanisms of mass loss due to roughness of even on smooth surface are complicated and detailed analyses are left for future work. For smooth and primed surfaces, the relationships proposed by Bretherton (1961) between liquid element velocity and film thickness could be used c.f.,(Bico and Quere 2001) or we could calculate surface porosity (or storativity) per unit fracture length defining an upper bound on rate of mass loss. Subsequent drainage and recapture of some of the mass stored on the surface by a stalled liquid element is plausible. Finally, although imbibition opportunity time scales are likely to be too short during bridge travel (perhaps analogous with evaporation from falling drops $\sim 3 \%$ mass loss over 10 's $\mathrm{m}$ ), the subsequent interactions of bridge lost mass (to roughness or priming) with rock matrix would clearly be affected by imbibition processes.

\section{Illustrative examples}

The foregoing theoretical considerations suggest that discrete liquid elements may travel relatively long distances within fractures even in the absence of hydraulic continuity (a prerequisite for application of continuum theories). The following examples illustrate the implications of such rapid motions and contrast them with commonly used continuum approximations. In the first example, we consider travel velocities of liquid bridges in fractures with impervious and smooth walls. The second example, illustrates the effect of surface porosity on the velocity of liquid bridges. The third 
example considers interaction of successive liquid bridges in fractures with significant surface porosity (storativity).

\subsection{Bridge velocity in impervious and smooth fracture surfaces}

The velocity of liquid bridges periodically released from an asperity or fracture discontinuity (relative to maximum velocity or the saturated hydraulic conductivity of the fracture) is described by (30). The results in Fig. 4(a) are comparisons of measured velocities of detached liquid bridges with calculated values from Eq. 30. We use measured data from Experiment I (Table 3) of Su et al. (2004). The measured quantities are: critical liquid bridge length $\left(L_{C}\right)$, length of detached liquid bridge $\left(L_{0}\right)$, fracture aperture $(b)$, fracture inclination angle $(\alpha)$, and velocity of detached liquid bridge $(U)$. In Fig. 4(b), the same experimental data were used to test the scaling law of Podgorski et al. (2001) given by Eq. 9. Considering experimental uncertainty, the results are in remarkable agreement with the postulated scaling law.

Equation 30 can be further reduced by substituting the definitions of $L_{C}$ and $L_{0}$ (Eqs. 14 and 34, respectively)

$$
\frac{U}{U_{\max }}=1-\frac{y_{0}(b)}{b} \frac{\cos \theta}{(\pi-2 \theta)} \Delta_{\theta}
$$

where $y_{0}(b)$ is as given by (2). In Fig. 5, we show the velocity of detached liquid bridge relative to the maximum for a wide range of fracture apertures calculated using Eq. 37. The general trend is that the length of detached liquid bridge relative to the stationary length increases with aperture. For fractures with relatively large aperture $(\sim 1 \mathrm{~mm})$, the initial length of liquid bridges is so large that their velocity is comparable to the saturated hydraulic conductivity. Such fast flow of discrete liquid clusters in the absence of hydraulic continuity is not expected to conform to current continuum approximations.

For quantitative comparison with continuum approximations, we focus our attention on two fracture apertures $(0.2 \mathrm{~mm}$ and $1 \mathrm{~mm})$, and critical contact angle hysteresis of $\Delta_{\theta}=0.15$ (comparable with the value of 0.15 calculated by Su et al. (2004) for glass surface). To establish an average travel velocity for a discrete liquid bridge traversing a fracture of length $\ell$, we need to account for both (a) bridge recharge time or the time interval between consecutive liquid bridge detachments, $\tau_{\mathrm{C}}$, and (b) actual travel velocity of the detached liquid bridge across the distance $\ell$. Using Eqs. 7 and 37, the average velocity is calculated as the ratio fracture length $\ell$ to the sum of detachment time $\tau_{\mathrm{C}}$ and post-detachment travel time $\ell / U$, yielding

$$
\bar{U}=\frac{U \ell}{\ell+\tau_{\mathrm{C}} U}
$$

The equivalent continuum approximation requires that the amount of mass flows as a continuous liquid film or thread flowing along the fracture. Hence, instead of liquid clusters, we consider films of uniform thickness flowing on both sides of a fracture. We make a conservative assumption that the width of the continuous film (thread) is equivalent to the width of the asperity supporting the liquid bridge $\left(y_{f} \geq y_{0}\right)$. Accordingly, the thickness of the falling film $h_{f}$ is related to the volumetric flux $Q$ by (note 

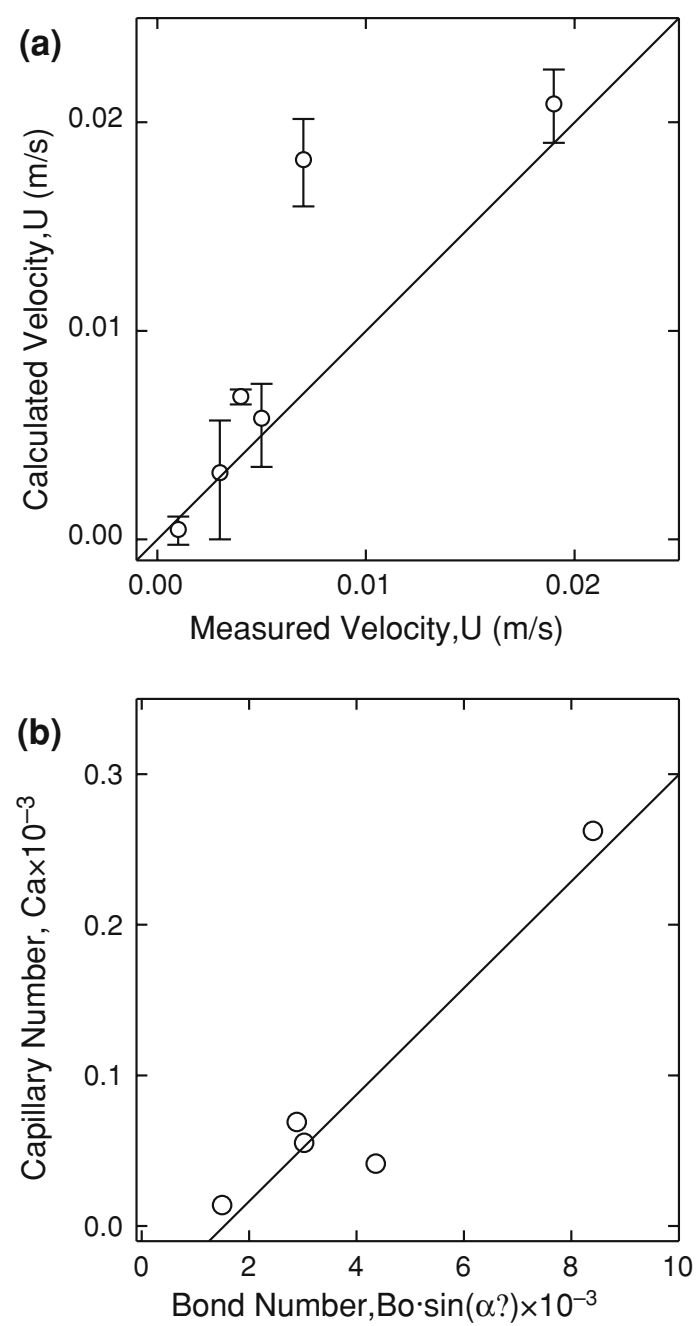

Fig. 4 (a) Comparison of measured versus calculated velocities of liquid bridges on smooth and rough glass surfaces; and (b) evaluation of the scaling law equation (9). Measurements and parameters obtained from Experiment I of Su et al. (2004)

that film flow on both fracture walls is considered)

$$
h_{f}=\left(\frac{3}{2} \frac{Q \eta}{y_{f} \rho g \sin \alpha}\right)^{3 / 2}
$$

and the average velocity of the film is:

$$
U_{f}=\frac{Q}{2 y_{f} h_{f}}
$$

In Fig. 6, we compare the dimensionless velocities of discrete liquid clusters with their equivalent continuous film for two fracture apertures over a wide range of volumetric fluxes. Generally, the results indicate that transport via discrete discontinuous 


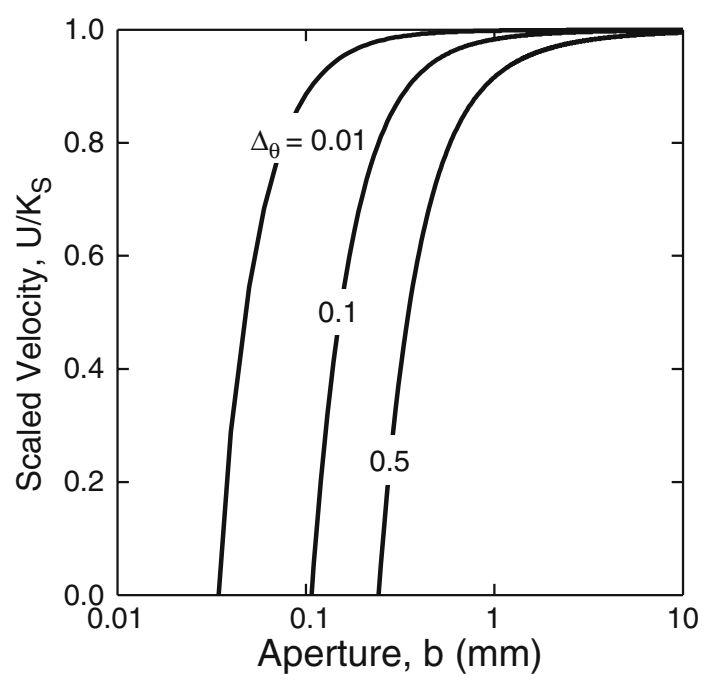

Fig. 5 Relative velocity of traveling liquid bridges for a wide range of fracture apertures and three values of $\Delta_{\theta}(0.01,0.1$, and 0.5$)$. Note that different values of $\Delta_{\theta}$ result in different critical lengths at detachment with associated different velocities

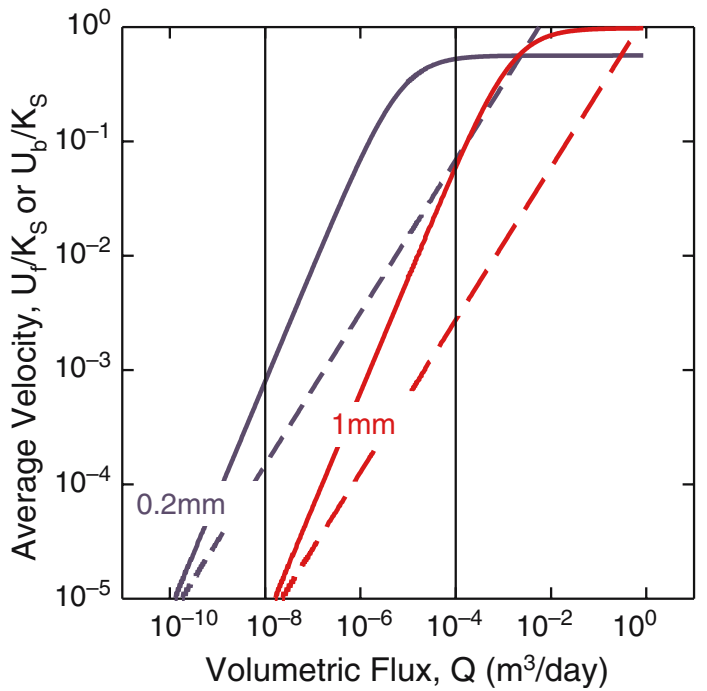

Fig. 6 Average velocity of traveling liquid bridges (lines) and film (dashed lines) for a wide range of volumetric fluxes and fracture apertures of $0.2 \mathrm{~mm}$ (blue lines) and $1 \mathrm{~mm}$ (red lines). The vertical lines (at fluxes of $10^{-8} \mathrm{~m}^{3} /$ day and $10^{-4} \mathrm{~m}^{3} /$ day) mark values used for comparison of distance of liquid bridges and film (see Fig. 7)

liquid clusters is much faster than continuous film flow. The disparity between the continuum and discrete flows vanishes for fractures near saturation or very dry conditions. Close to saturation, the velocity of thick films approaches maximum velocity of infinitely long liquid bridge (also equals the saturated hydraulic conductivity of the fracture under unit hydraulic gradient). 


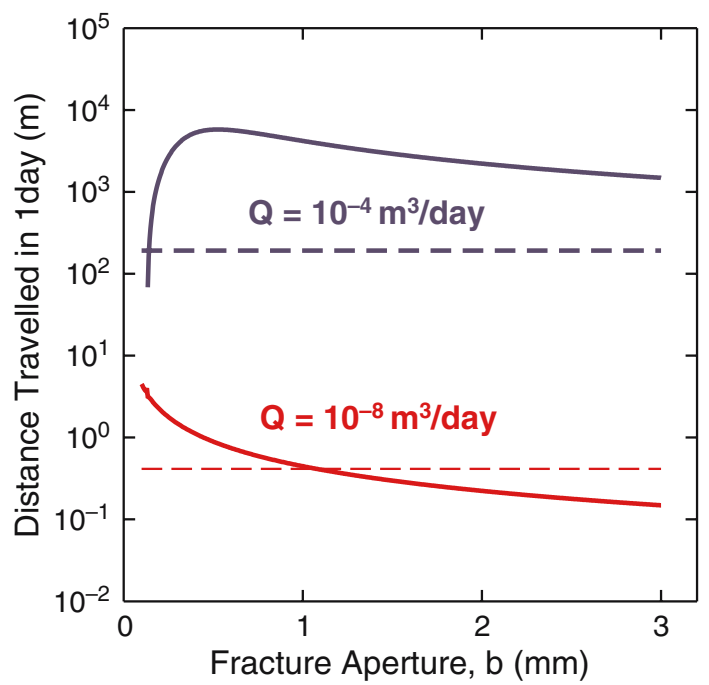

Fig. 7 Comparison of distance traveled by liquid bridges (solid lines) and film (dashed lines) down a fracture in one day, assuming an infinitely long fracture and constant flow rates of $10^{-8} \mathrm{~m}^{3} / \mathrm{day}$ (red lines) and $10^{-4} \mathrm{~m}^{3} /$ day (blue lines) (see Fig. 6) along a $1 \mathrm{~cm}$ wide fracture section

To examine the consequences of such rapid motions on travel distances, we evaluate the distance traveled by liquid bridges and steady film over the course of one day for two hypothetical steady fluxes of $10^{-4} \mathrm{~m}^{3} /$ day and $10^{-8} \mathrm{~m}^{3} /$ day within a hypothetical vertical fracture of unlimited length and width of $1 \mathrm{~cm}$. The results summarized in Fig. 7 , show that for narrow aperture fractures, discrete liquid bridges traverse considerably larger distances than traveled by continuous film flow (which does not depend on aperture size). The increase in flux from $10^{8} \mathrm{~m}^{3} /$ day and $10^{-4} \mathrm{~m}^{3} /$ day results in remarkable enhancement in travel distances even for fractures with larger aperture sizes. The results provide a vivid illustration of the enhancement in travel velocity and distance due to formation of discrete liquid elements in fractured rock. We note that the primary effect of such focused transport mechanisms is not necessarily enhanced transport of more liquid mass, but the potential for more rapid arrival times of pollutants carried with such discrete liquid bridges.

The above example depicts maximum disparity between continuum flow and discrete flow without retardation. In the next subsection, we illustrate how discrete bridges interact with porous and/or rough surfaces.

\subsection{Effect of surface porosity on velocity of liquid bridges}

The distance traveled by liquid-bridges (33) released from an asperity is shown in Fig. 8 for two different values of surface porosity $\left(\zeta=2 \times 10^{-6} \mathrm{~m}^{3} \mathrm{~m}^{-2}\right.$ and $\zeta=$ $\left.10^{-5} \mathrm{~m}^{3} \mathrm{~m}^{-2}\right)$ as well as a smooth and impervious fracture surface $\left(\zeta=0 \mathrm{~m}^{3} \mathrm{~m}^{-2}\right)$ with no mass loss. Higher surface porosity leads to rapid slowdown and short travel distance. In smooth walled fractures, a single liquid bridge detachment event is sufficient to traverse a fracture of any length. Whereas rough or porous fracture surface requires interaction between multiple detachment events for flow across a fracture. 

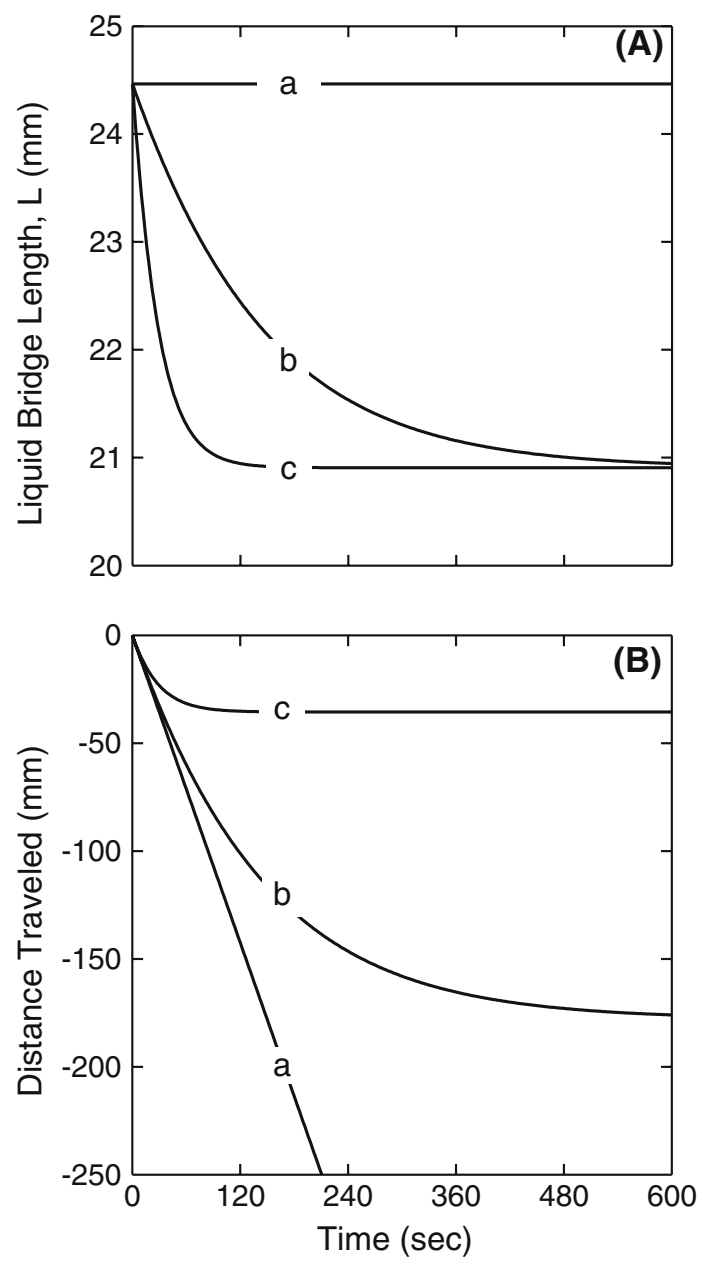

Fig. 8 Length (a) and distance traveled (b) by liquid bridges formed in a fracture of aperture $b=0.1 \mathrm{~mm}\left(L_{0}=24.5 \mathrm{~mm}\right.$ initial velocity $\left.1.2 \mathrm{~mm} / \mathrm{s}\right)$ with surface porosities of $i=0 \mathrm{~m}^{3} \mathrm{~m}^{-2}, i i=$ $2 \times 10^{-6} \mathrm{~m}^{3} \mathrm{~m}^{-2}$, and $i i i=10^{-5} \mathrm{~m}^{3} \mathrm{~m}^{-2}$

In the next subsection, we consider such interaction between successive detachment events using a few simple assumptions about the surface porosity.

\subsection{Interaction between multiple traveling bridges}

Here we are concerned with interactions among successive liquid bridges formed at the location and follow a similar path (Ghezzehei and Or 2005). We track each liquid bridge from detachment as it travels down the fracture while losing mass and momentum. When a traveling liquid bridge merges with a stalled preceding bridge, the aggregate mass travels at a higher velocity until it loses sufficient mass and stalls. The initial length of a liquid bridge departing from an asperity, $L_{0}=L(t=0)$, is obtained from Eq. 34 and Ghezzehei and Or (2005). Following coalescence of two or 
more, the resulting length of the newly formed liquid bridge is related to volume of coalesced liquid bridges by

$$
L_{0}=\frac{\sum_{i} V_{i}}{\rho b y_{0}}, \quad t=0
$$

where $V_{i}$ stands for the combined volume of the coalescing bridges.

The passage of liquid bridges leaves behind a trail of partially filled depressions (or partially saturated exposed pores) with reduced wicking ability. As the retained liquid drains or is transmitted deeper into the matrix, the wicking ability of the fracture surface (surface porosity) is recovered gradually. For simplicity, the recovery of the surface porosity is modeled here by exponential function of time

$$
\zeta=\zeta_{o}[1+\exp (-\kappa t)]
$$

where $\zeta_{o}$ is the surface porosity of dry surface and $\kappa$ is the rate of recovery, which is related to how fast the trapped liquid can be absorbed by the matrix or drain down as film. Consequently, it is a measure of matrix imbibition or film flow. As the fracture surface becomes wet with each passing liquid bridge, the rate at which it dries decreases, reflected in decrease in $\kappa$. The change in $\kappa$ at any given location is related to the frequency of liquid bridge passage through that location and could be scaled as

$$
\kappa \sim \kappa_{O} / N^{\tau}
$$

where $\kappa_{o}$ is the rate of recovery after the first liquid bridge has passed through a point and $N$ is the number of the liquid bridge passing through a given point. An illustrative example of the rate of surface porosity recovery $(\zeta)$ as a function of time is shown for the first few liquid bridges in Fig. 9.

In Fig. 10, we illustrate the steps involved in coalescence of pairs of primary liquid bridges (formed directly underneath asperities) to form secondary liquid bridges,

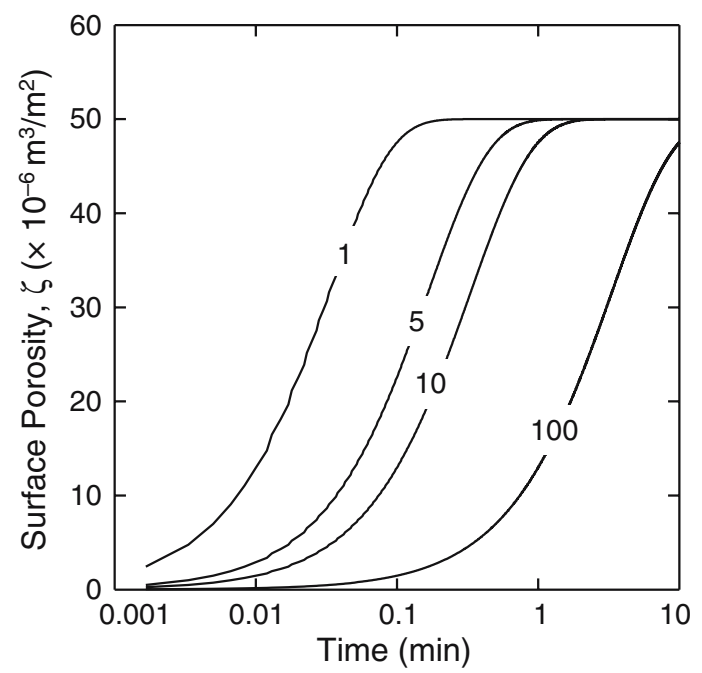

Fig. 9 Change in effective surface porosity after passage of $N$ th liquid bridge for $N=1,5,10$, and 100 , where initial porosity is $\zeta_{0}=50 \times 10^{-6} \mathrm{~m}^{3} \mathrm{~m}^{-2}$ 


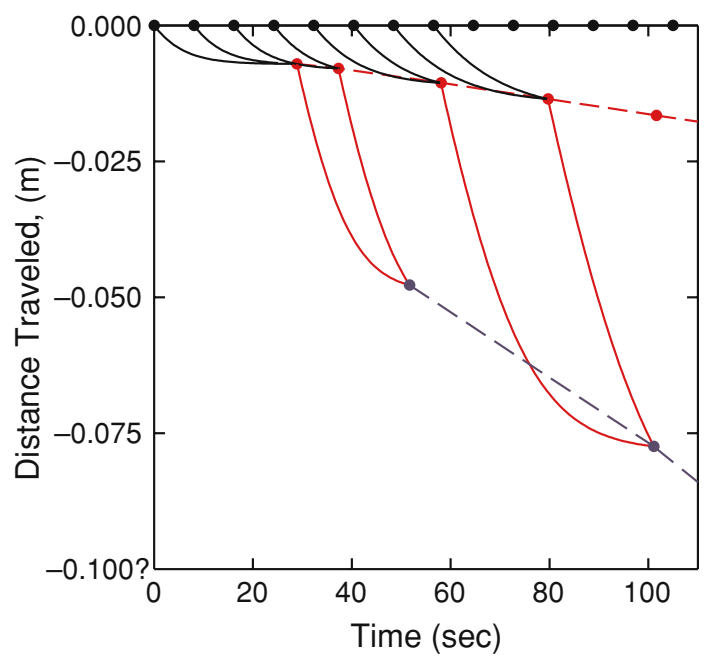

Fig. 10 Pathways of liquid bridges formed in a $b=0.1 \mathrm{~mm}$ aperture fracture, at flux of $Q=2 \mathrm{ml} / \mathrm{h}$. Surface porosity is $\zeta_{0}=50 \times 10^{-6} \mathrm{~m}^{3} \mathrm{~m}^{-2}$ and a rate of $\kappa=0.5 \mathrm{~s}^{-1}$

followed by coalescence of a pair of secondary bridges to form tertiary liquid bridge. The increased mass with each coalescence is followed by faster and longer travel distance before mass loss slows down the liquid bridge. Obviously, mass accumulation into a single bridge is not unlimited and more complexities and modes of fragmentation are expected and the process cascades down the fracture (Podgorski et al. 2001).

It is interesting to consider extension of above results by forming zones signifying different time and distance relationships for liquid bridges traveling in rough-walled fracture relative to behavior of non-retarded liquid bridge (Fig. 11). Interactions between multiple liquid bridges resulting from slowdown amplify liquid bridge size that enhances overall travel velocity beyond that of liquid bridges traveling in a smooth fracture of the same nominal aperture. For example, the quaternary liquid bridges have higher velocity (denoted by the slopes in Fig. 11) than the liquid bridges traveling along smooth fractures. Consequently, the travel distance predictions presented in Fig. 7 should be considered lower bound of the transport capacity in rough-walled fractured rock.

\section{Summary and conclusions}

The study focuses on behavior of discrete liquid elements that form, detach, and travel in unsaturated fractures. Discrete elements and associated intermittent flow regimes present a challenge to standard continuum models, and may represent a mode for rapid mass transport across considerable distance in formations considered hydraulically inactive. Extending the results of Ghezzehei and Or (2005) on formation and detachment of liquid bridges, we first analyzed the conditions for onset of motion for simple 1-D and ellipsoidal liquid elements. The results relating gravitational forces to pinning capillary forces follow a scaling law proposed by Podgorski et al. (2001) that 


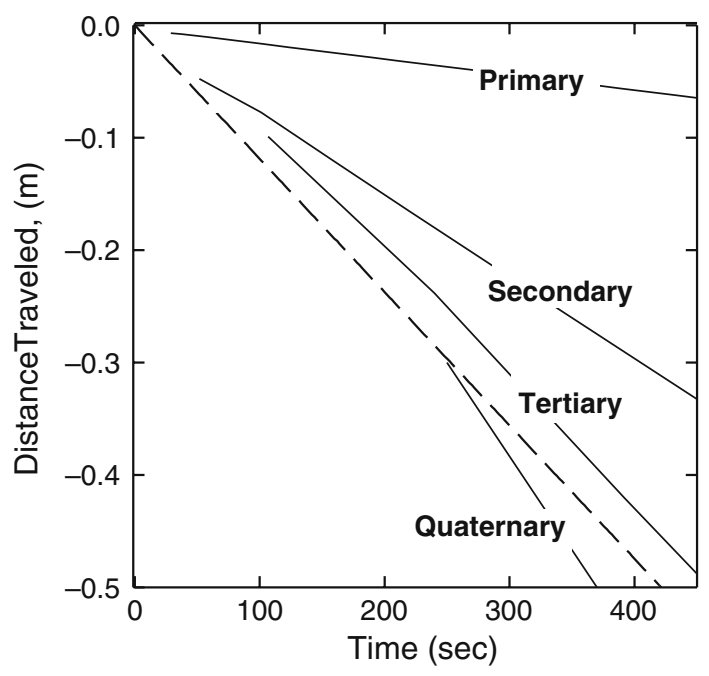

Fig. 11 Zones of different time and distance relationships for liquid bridges traveling in rough-walled fracture. Dashed line represents the path of liquid bridge released at $t=0$ and traveling in smooth fracture. Fracture properties are identical to Fig. 10

offers a general and unifying framework for the interplay between capillary, gravity and viscous forces governing motion of discrete liquid elements in a range of capillary conduits.

The introduction of permeability for the specific geometry and the threshold for onset of motion enable prediction of steady velocity of discrete liquid elements in fractures (and other systems). Interactions with fracture walls due to loss of mass, either due to surface priming, or liquid retention in surface roughness, give rise to complex travel velocity-distance relationships. Surprisingly, rougher surfaces that tend to retard motions of all liquid bridges, may promote coalescence of multiple bridges and in effect accelerate the rate of mass transport across larger distances. Illustrative examples of travel distances and mass for different fracture roughness characteristics were presented. The potential for liquid absorption into the rock or film drainage downstream on temporal changes in surface retention capacity (hence bridge travel behavior) were explored in generic (parametric) form.

Comparisons of discrete versus continuum transport mechanisms in unsaturated fractured media reveal large disparity between rapid travel velocities and larger distances by discrete liquid elements relative to their continuum film flow counterpart. The consequences of such transport mechanisms with the potential for focusing of diffuse liquid films to rapidly traveling discrete liquid bridges should be factored into waste isolation designs and water resource risk assessment. Such flow focusing processes may be amplified considering a population of liquid bridges within a fracture plane and mass accumulation in fracture intersections. The results provide a useful framework for addressing and perhaps quantifying complex and intermittent flow regimes observed in field and laboratory experiments. Limitations related to internal fracture geometry and surface properties, interactions within fractures, and other assumptions will be explored in future experimental and theoretical studies. 


\section{Appendix 1: Equivalence of the scaling law of Podgorski et al. (2001) with energy dissipation equations}

In this appendix we derive the scaling law of Podgorski et al. (2001) from considerations of general energy dissipation equations. Experiments of drops sliding on smooth surfaces have shown that moving drops are parallel-sided (Bikerman 1950; Furmidge 1962; Larkin 1967). We assume these observations also hold for liquid bridges in fractures (Fig. 2a). For the remainder of this appendix, we assume that the advancing and receding edges are approximated by semicircles of diameter $D$ and have a rectangular center of length $D \beta$, as shown in Fig. $2 \mathrm{~d}$. The total length of a liquid bridge is then $L=D(1+\beta)$. Then, the volume of the moving liquid bridge in a fracture with aperture $b$ is

$$
V=b D^{2}\left(\frac{\pi}{4}+\beta\right)
$$

The profiles of the advancing and receding menisci are described in polar coordinates by $(r, \varphi)$, where $0 \leq \varphi \leq \pi / 2$ for the receding edge and $\pi / 2 \leq \varphi \leq \pi$ for the advancing edge (Fig. 2c). By symmetry, we only consider half of the liquid bridge and use a factor of two to account for the other half.

\section{A.1 Energy balance equation}

Consider a liquid bridge sliding down a fracture at constant velocity $U$. The gravitational potential energy decreases at a constant rate of

$$
\Phi_{G}=U V \rho g \sin \alpha
$$

This decrease in energy is consumed by (1) dissipation due to motion of the solidliquid-vapor contact line, $\Phi_{L}$ and (2) viscous dissipation due to velocity field of the liquid bridge, $\Phi_{V}$ (de Gennes 1985):

$$
\Phi_{G}=\Phi_{L}+\Phi_{V}
$$

Other forms of energy dissipation, such as rolling motion of liquid at the peripheries of the bridge (Huh and Scriven 1971) and flow of precursor and trailing films, are assumed to play negligible roles.

\section{A.2 Energy dissipation by moving contact line}

The rate of energy dissipation due to wetting (dewetting) along an infinitesimal segment of the contact line $\mathrm{d} S$ is

$$
\mathrm{d} \phi_{L}=q U_{n} \mathrm{~d} S
$$

where $U_{n}=U \cos \varphi$ is the velocity component normal to the contact line (positive in the direction of motion), and $q$ is the work done in moving the contact line by a unit displacement per unit segment of the contact line and is described by the Young-Dupre equation

$$
q=\sigma_{L V}\left(\cos \theta-\cos \theta_{0}\right)
$$

We make a simplifying assumption that the contact angle for the advancing edge is constant at the critical value of $\theta_{\mathrm{A}}$ and for the receding edge at $\theta_{\mathrm{R}}$. The consequences 
of this simplifying assumption are discussed later. Because the sides of the moving bridge are parallel to the direction of flow, they do not contribute to energy is loss or gain during motion. The total rate of energy loss due to motion of the contact line of a liquid bridge is determined from integration along the receding and advancing contact lines,

$$
\begin{aligned}
\Phi_{L} & =-4 \sigma_{L V} U \frac{D}{2}\left\{\int_{0}^{\pi / 2}\left(\cos \theta_{\mathrm{a}}-\cos \theta_{0}\right) \cos \varphi \mathrm{d} \varphi+\int_{0}^{\pi / 2}\left(\cos \theta_{\mathrm{r}}-\cos \theta_{0}\right) \cos \varphi \mathrm{d} \varphi\right\} . \\
& =2 \sigma_{L V} U\left(\cos \theta_{\mathrm{r}}^{*}-\cos \theta_{\mathrm{a}}^{*}\right)
\end{aligned}
$$

where the factor of four accounts for the two sides of the fracture walls and the symmetric half of the bridge and $\mathrm{d} \varphi=D \mathrm{~d} S / 2$. Note that wetting consumes energy and dewetting releases energy.

\section{A.3 Viscous dissipation}

The viscous dissipation arises from Poiseuille type flow of the liquid bridge. Then, the flow velocity at a point and the average velocity parallel to the major gravitational gradient are scaled as, respectively

$$
\begin{gathered}
u(z) \sim\left(z^{2}-b^{2} / 4\right) \\
U \sim b^{2} / 6
\end{gathered}
$$

The rate of viscous dissipation per an infinitesimal unit volume of laminar unidirectional flow of the liquid bridge is given by Lamb (1945)

$$
\phi_{V}=\eta\left(\frac{\partial u}{\partial z}\right)^{2}
$$

Assuming the velocity distribution given by (51) holds for the entire liquid bridge (including the curved menisci at the edges), the total viscous dissipation is given integrating equation (52) over the entire volume of the bridge,

$$
\Phi_{V}=\eta \int_{V}\left(\frac{\partial u}{\partial z}\right)^{2} \sim \eta V\left(\frac{3 U}{b}\right)^{2}
$$

where $V$ is the total volume given by Eq. 44, and we used Eqs. 50 and 51 to derive the following scaling

$$
\frac{2}{b} \int_{0}^{b / 2}\left(\frac{\partial u}{\partial z}\right)^{2}=\frac{b}{2}=\frac{3 U}{b}
$$

\section{A.4 Sliding velocity}

At steady state we equate the sum of the energy dissipation rate (Eqs. 49, 53) with the rate of decrease in potential energy (Eq. 45) leading to

$$
U D \eta \frac{9}{b^{2}} \sim-\frac{4}{\pi+4 \beta} \frac{\sigma}{b}\left(\cos \theta_{\mathrm{r}}^{*}-\cos \theta_{\mathrm{a}}^{*}\right)+\rho g b D \sin \alpha
$$


Recalling that the length of liquid bridge is $L=D(1+\beta)$, (55) can be further simplified as,

$$
\mathrm{Ca} \sim \frac{b}{9 L}\left[\mathrm{Bo} \sin \alpha-\frac{4(1+\beta)}{\pi+4 \beta} \Delta_{\theta}\right]
$$

where $\mathrm{Ca}=\eta U / \sigma, \mathrm{Bo}=\rho g b L / \sigma$ and $\Delta_{\theta}=\cos _{\mathrm{r}}^{*}-\cos _{\mathrm{a}}^{*}$. Equation 56 is equivalent to the steady velocity expression given by Eq. (28) and is the scaling law of Podgorski et al. (2001) given in (9).

\section{References}

Bico, J., Quere, D.: Falling slugs. J. Colloid Interface Sci. 243(1), 262-264 (2001)

Bikerman, J.J.: Sliding of drops from surfaces of different roughnesses. J. Colloid Sci. 5(4), 349-359 (1950)

Bretherton, F.P.: The motion of long bubbles in tubes. J. Fluid Mech. 10(2), 166-188 (1961)

de Gennes, P.G.: Wetting: Statics and dynamics. Rev. Mod. Phys. 57(3), 827-863 (1985)

Extrand, C.W., Kumagai, Y.: Liquid-drops on an inclined plane - the relation between contact angles, drop shape, and retentive force. J. Colloid Interface Sci. 170(2), 515-521 (1995)

Faybishenko, B., Bodvarsson, G.S., Salve, R.: On the physics of unstable infiltration, seepage, and gravity drainage in partially saturated tuffs. J. Contaminant Hydrol. 62(3), 63-87 (2003)

Faybishenko, B., Doughty, C., Steiger, M., Long, J.C.S., Wood, T.R., Jacobsen, J.S., Lore, J., Zawislanski, P.T.: Conceptual model of the geometry and physics of water flow a fractured basalt vadose zone. Water Resour. Res. 36(12), 3499-3520 (2000)

Furmidge, C.G.: Studies at phase interfaces .1. Sliding of liquid drops on solid surfaces and a theory for spray retention. J. Colloid Sci. 17(4), 309 (1962)

Ghezzehei, T.A., Or, D.: Liquid fragmentation and intermittent flow regimes in unsaturated fractured porous media. Water Resour. Res. 41(12), Art. No. W12406, (2005)

Glass, R.J., Nicholl, M.J., Ramirez, A.L., Daily, W.D.: Liquid phase structure within an unsaturated fracture network beneath a surface infiltration event: field experiment - art. No. 1199. Water Resour. Res. 38(10), 1199 (2002)

Ho, C.K.: Asperity-induced episodic percolation in channels and fractures. J. Porous Media 7(3), 155-164 (2004)

Hoffman, R.L.: A study of the advancing interface. I. Interface shape in liquid-gas systems. J. Colloid Interface Sci. 50(2), 228-241 (1975)

Huh, C., Scriven, L.E.: Hydrodynamic model of steady movement of a solid/liquid/fluid contact line. J. Colloid Interface Sci. 35(1), 85-101 (1971)

Kim, H.-Y., Lee, H.J., Kang, B.H.: Sliding of liquid drops down an inclined solid surface. J. Colloid Interface Sci. 247(2), 372-380 (2002)

Lamb, H.: Hydrodynamics. Dover, New York, NY (1945)

Larkin, B.K.: Numerical solution of the equation of capillarity. J. Colloid Interface Sci. 23(3), 305-312 (1967)

Meheust, Y., Lovoll, G., Maloy, K.J., Schmittbuhl, J.: Interface scaling in a two-dimensional porous medium under combined viscous, gravity, and capillary effects. Phys. Rev. E 66(5), Art. No. 051603 (2002)

Nicholl, M.J., Glass, R.J., Wheatcraft, S.W.: Gravity-driven infiltration instability in initially dry nonhorizontal fractures. Water Resour. Res. 30(9), 2533-2546 (1994)

Persoff, P., Pruess, K.: Two-phase flow visualization and relative permeability measurement in natural rough-walled rock fractures. Water Resour. Res. 31(5), 1175-1186 (1995)

Podgorski, T., Flesselles, J.M., Limat, L.: Corners, cusps, and pearls in running drops - art. No. 036102. Phys. Rev. Lett. 8703(3), 6102-NIL_95 (2001)

Prazak, J., Sir, M., Kubik, F., Tywoniak, J., Zarcone, C.: Oscillation phenomena in gravity-driven drainage in coarse porous-media. Water Resour. Res. 28(7), 1849-1855 (1992)

Spiegel, M.R., Liu, J.: Mathematical Handbook of Formulas and Tables, 278 pp. McGraw-Hill Companies Inc., New York (1999)

Su, G.W., Geller, J.T., Hunt, J.R., Pruess, K.: Small-scale features of gravity-driven flow in unsaturated fractures. Vadose Zone J. 3(2), 592-601 (2004)

글 Springer 
Su, G.W., Geller, J.T., Pruess, K., Wen, F.: Experimental studies of water seepage and intermittent flow in unsaturated, rough-walled fractures. Water Resour. Res. 35(4), 1019-1037 (1999)

Wilson, S.D.R.: The slow dripping of viscous fluid. J. Fluid. Mech. 190, 561-570 (1988)

Wood, T.R., Glass, R.J., McJunkin, T.R., Podgorney, R.K., Laviolette, R.A., Noah, K.S., Stoner, D.L., Starr, R.C., Baker, K.: Unsaturated flow through a small fracture-matrix network: Part 1. Experimental observations. Vadose Zone J. 3(1), 90-100 (2004) 